\title{
The physics of the centrality dependence of elliptic flow
}

\author{
S.A. Voloshin ${ }^{(a, b)}$ and A.M. Poskanzer ${ }^{(a)}$ \\ (a) Nuclear Science Division, Lawrence Berkeley National Laboratory, Berkeley, California 94720 \\ (b) Department of Physics and Astronomy, Wayne State University, Detroit, MI 48201
}

(June 25, 2018)

\begin{abstract}
The centrality dependence of elliptic flow and how it is related to the physics of expansion of the system created in high energy nuclear collisions is discussed. Since in the hydro limit the centrality dependence of elliptic flow is mostly defined by the elliptic anisotropy of the overlapping region of the colliding nuclei, and in the low density limit by the product of the elliptic anisotropy and the multiplicity, we argue that the centrality dependence of elliptic flow should be a good indicator of the degree of equilibration reached in the reaction. Then we analyze experimental data obtained at AGS and SPS energies. The observed difference in the centrality dependence of elliptic flow could imply a transition from a hadronic to a partonic nature of the system evolution. Finally we exploit the multiplicity dependence of elliptic flow to make qualitative predictions for RHIC and LHC.
\end{abstract}

The goal of the ultrarelativistic nuclear collision program is the creation of the QGP quark-gluon plasma - the state of deconfined quarks and gluons. It is understood that such a state requires (local) thermalization of the system brought about by many rescatterings per particle during the system evolution. It is not clear when and if such a dynamical thermalization can really occur. An understanding of these phenomena can be achieved by considering elliptic flow [1] recently studied at AGS [2] and SPS [3] energies. It will be shown how the centrality dependence of the strength of elliptic flow, $v_{2}$, defined as the second coefficient in the Fourier decomposition of the particle azimuthal distribution [4], is an indicator of the degree of equilibration (thermalization) achieved in the system. 
Our qualitative conclusions are based on the observation, that in the hydro limit (which we equate in our discussion to complete thermalization) and in the opposite limiting case, the low density limit, (where dynamical thermalization is not expected) the centrality dependence of elliptic flow is different. In the hydro limit, the mean free path is much less than the geometrical size of the system. The centrality dependence of flow is totally governed in this case by the initial geometry (eccentricity), the latter being roughly proportional to the impact parameter. In the low density limit, the mean free path is comparable to or larger than the system size. The final anisotropy in this case should be proportional to the ratio of the system size to the mean free path (the number of collision). The anisotropy vanishes in the limit of infinite mean free path. The latter in its turn depends on the particle density, which is largest for central collisions and vanishes for very peripheral collisions. Note that the factors involved change drastically with centrality. One could imagine other reasons for centrality dependence of elliptic flow in the hydro model, such as the initial conditions, viscous corrections, resonances, or effective volume corrections, but we expect that all these other factors have a much weaker dependence on the impact parameter. By considering the two limiting cases we hope to highlight qualitative considerations important for understanding the degree of thermalization and the partonic or hadronic nature of the collisions. In essence we present a framework for examining these questions experimentally, however at the moment, it is mainly the experimental data which are not adequate to answer these questions convincingly.

We will often use the term "physics of the collision". By this we mean both the degree of equilibration and whether the hadronic picture in terms of nucleons, pions, etc., or the partonic picture in terms of deconfined quarks and gluons, is more applicable to the evolution of the system. The partonic picture in our view is similar to a QGP but the system is not necessarily thermalized.

\section{Low Density Limit}

To discuss the centrality dependence of $v_{2}$ more quantitatively, we start from the hypothesis that the system is not dense and its evolution can be described by the first correction 
to the collisionless limit [5]. Physically this means that the rescattering occurring during the system evolution changes the particle momenta very little on the average and the corresponding change in the distribution functions can be treated in first order as perturbations. Under this assumption the final elliptic flow, $v_{2}$, is proportional to the initial overlapping region elliptic anisotropy, $\varepsilon$, (introduced in flow analyses in [1] and in its present form in [5, 6]) and to the initial particle space density which defines the probability of particles to rescatter [5.

The initial geometry of the overlapping zone can be evaluated in a simple Glauber type model with a Woods-Saxon nuclear density. The results are weakly dependent on the weights used [7]. What is important is that if one wants to compare different energies, e.g. AGS, SPS and RHIC, the nuclear geometry cancels out, and only the dependence on multiplicity is left. This is true provided that the "physics" of of the system evolution stays the same. If it changes then the scaling with multiplicity will be violated. This is a very important point if one reads it the other way around: if scaling is not observed then probably the physics has changed.

Under the assumption that the system is relatively dilute the momentum anisotropy is proportional to the spatial anisotropy, but also the particles must scatter to probe that anisotropy. Thus, the spectra distortion is directly proportional to the spatial anisotropy and the number of rescatterings, or the particle density in the transverse plane. In this limit the final elliptic flow (see a more detailed formula in [5])

$$
v_{2} \propto \varepsilon \frac{1}{S} \frac{d N}{d y},
$$

where $S=\pi R_{x} R_{y}$ is the area of the overlapping zone, with $R_{x}^{2} \equiv\left\langle x^{2}\right\rangle$ and $R_{y}^{2} \equiv\left\langle y^{2}\right\rangle$ describing the initial geometrical sizes of the system in $x$ and $y$ directions, respectively. (The $\mathrm{x}-\mathrm{z}$ axes lie in the reaction plane). The averages include a weighting with the number of collisions along the beam axis. The initial space elliptic anisotropy is defined as

$$
\varepsilon=\frac{R_{y}^{2}-R_{x}^{2}}{R_{x}^{2}+R_{y}^{2}} .
$$


In our calculation we use a Woods-Saxon parameterization of the nuclear density with parameters $R_{A}=1.12 \cdot A^{1 / 3}$, and $a=0.547 \mathrm{fm}$. More information on the effect of different weights and the values of $R_{x}^{2}, R_{y}^{2}, S$ and $\varepsilon$ as a function of impact parameter can be found in [7]. The proportionality coefficient in Eq. (1) is defined by the "physics" of the rescattering. If the physics is the same in central and peripheral collisions then Eq. (1) yields the centrality dependence of $v_{2}$.

\section{Hydro Limit}

As follows from Eq. (1) the elliptic flow increases with the particle density. Eventually it will saturate [8] at the hydro limit, which would mean complete thermalization of the system. In this regime the centrality dependence of elliptic flow is mainly determined by the initial elliptic anisotropy of the overlapping zone in the transverse plane [8], and the ratio of the two should be approximately constant as shown in the first such calculations done by Ollitrault [1]. From his results it follows that $\left(v_{2} / \varepsilon\right)_{\text {hydro }} \approx 0.27-0.35$, depending on the equation of state used (with or without QGP)]. The calculations [9] give a somewhat smaller flow, resulting in $\left(v_{2} / \varepsilon\right)_{h y d r o} \approx 0.21-0.23$ (partly due to the realistic treatment of resonances which decrease the pion flow by about 15\%). Note that in both calculations, [1] and [9], the longitudinal expansion of the system is treated analytically assuming Bjorken scaling. Real 3D hydro calculations would be very useful, although we do not expect that

\footnotetext{
${ }^{1}$ To avoid confusion, note the difference in definitions of $\varepsilon$ used in Eq. (2) of this paper and $\alpha_{x}$ from [1]. For $\mathrm{Pb}+\mathrm{Pb}$ collisions the maximal value of $\varepsilon \approx 0.44$ compared to $\alpha \approx 0.3$.. Then, the results [1] yield $v_{2}^{\left\{p_{t}^{2}\right\}} / \varepsilon \approx 0.55-0.7$, where $v_{2}^{\left\{p_{t}^{2}\right\}}$ means the elliptic flow weighted with $p_{t}^{2}$. Recent calculations [9] show that the particle elliptic flow is related to this quantity as $v_{2} \approx 0.5 v_{2}^{\left\{p_{t}^{2}\right\}}$.
} 
they would greatly change the centrality dependence.

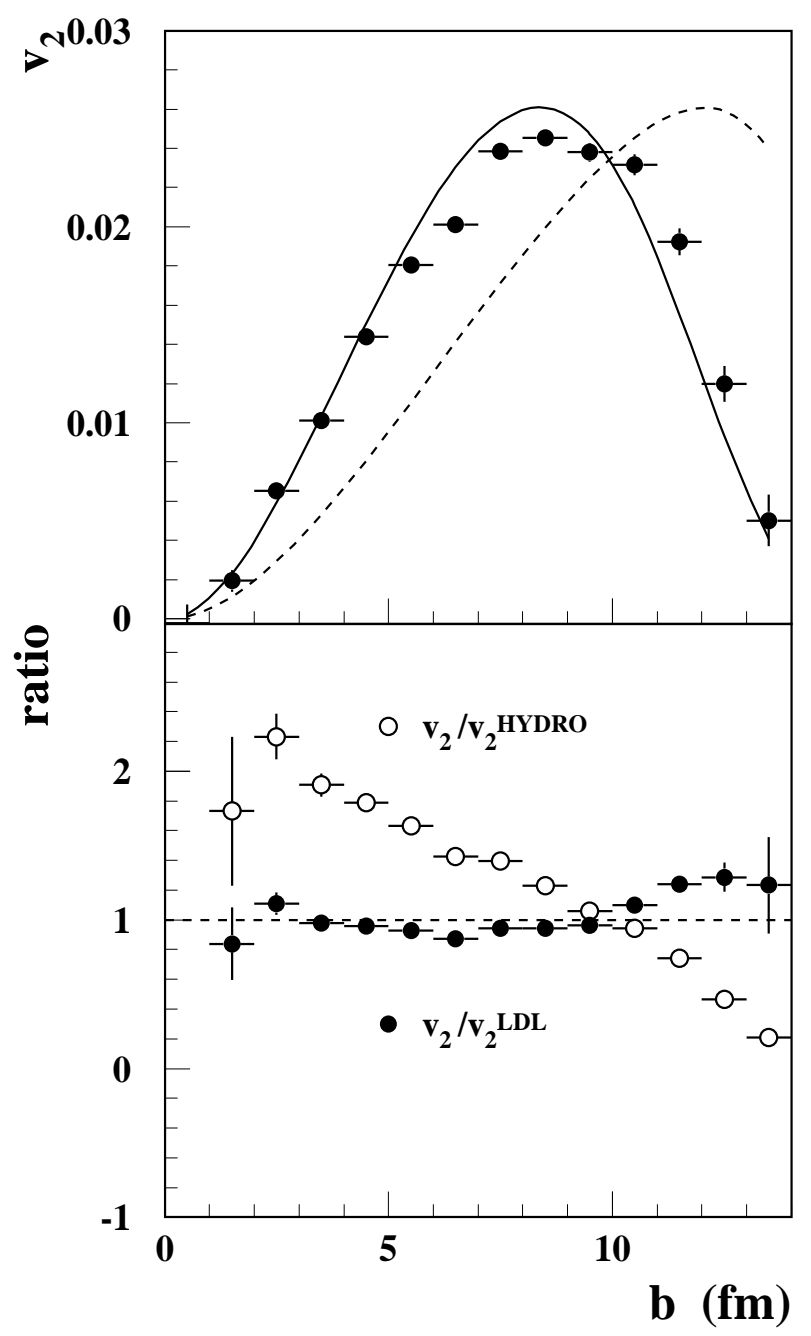

FIG. 1. Top: comparison of elliptic flow, $v_{2}$, for pions from RQMD ver. 2.3 (filled circles) with the dependence expected for the low-density limit (solid line) and that expected for the hydro limit (dashed line). Bottom: ratios of $v_{2} / v_{2}^{L D L}$, and $v_{2} / v_{2}^{H Y D R O}$.

\section{RQMD}

Before discussing the experimental data we will first consider a realistic model. We take RQMD v2.3 [10] for our calculations. Fig. 1 top shows the comparison of the directly calculated $v_{2}$ of pions in $\mathrm{Pb}+\mathrm{Pb}$ collisions at $158 \mathrm{GeV} \cdot \mathrm{A}$ collisions at mid-rapidity $(-1<$ $y<1$ ) with the expectation from the low density limit, $v_{2}^{L D L}$ (Eq. (1) normalized to the same area under the curve in order to illustrate just the centrality dependence.) One can 
see rather good agreement, which suggests that RQMD is close to the low density limit even as one scans the centrality from peripheral to central collisions. (In this version of RQMD no QGP is simulated.) This is not that striking a conclusion, considering that no hydro-type behavior has ever been observed in RQMD. Note that the low density limit does not mean a low number of total rescatterings. The number of rescatterings can be large provided all of them are relatively soft and the particle momentum changes little compared to the initial momentum. The cross section which enters the equations is the transport (not total) cross section (see [5]). The centrality dependence expected for the hydro limit is shown on the same plot by a dashed line also normalized to the same area under the curve $\left(v_{2}^{H Y D R O} \approx 0.059 \varepsilon\right)$. Note the large difference between the two curves, which was not noted in [5]. Fig. 1 bottom shows that the ratio of $v_{2}$ to the expected functional form is flat for the low density limit but not for the hydro limit. A centrality dependence similar to the low density limit was also observed in [11] where a computer simulation of a pion gas expansion was studied.

\section{Data}

Now let us turn to the experimental data. At AGS energies the elliptic flow of charged particles and of transverse energy was measured by the E877 Collaboration. Unfortunately, the publication [2] containing the detailed pseudorapidity dependence for each centrality lacks a figure showing just the centrality dependence. Our estimates based on their data [2] 
of charged particle flow at midrapidity are presented in Fig. 2.

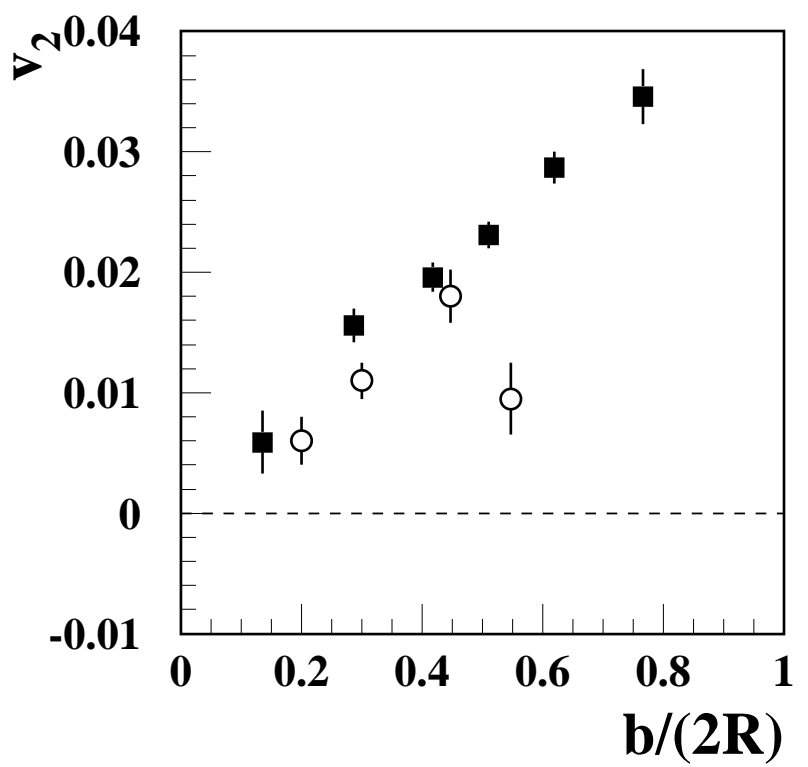

FIG. 2. Elliptic flow at the AGS (open circles) and the SPS (filled squares).

The data indicate that at AGS the flow peaks at mid-centralityf, consistent with the low density limit prediction and no change in physics with centrality. At this energy some decrease of elliptic flow in peripheral collisions can be also attributed to shadowing by spectator matter. At SPS [13], preliminary data indicate that the elliptic flow peak moves towards peripheral collisions. This fact itself would hint at the hydro-dynamical picture of the system evolution. A more detailed look at the data shows that this is unlikely. First, the maximal value of elliptic flow $\left(v_{2} \leq 0.04\right)$ is significantly less than predicted by hydro calculations [1,9] (about 0.09-0.1)․ Second, in the hydro limit elliptic flow should depend

\footnotetext{
${ }^{2}$ A similar centrality dependence of transverse energy flow (from the same data [2]) can be found in the thesis of Chang [12].

${ }^{3}$ In [9] agreement was claimed between hydro and the NA49 mid-central data [3] leading to their conclusion of complete equilibration. However, this comparison was done for $p_{t}<0.3 \mathrm{GeV} / \mathrm{c}$ and
} 
only on the initial space elliptic anisotropy, $\varepsilon$. The preliminary NA49 data indicate that the ratio $v_{2} / \varepsilon$, at least for semi-central collisions, is likely increasing with centrality 13 (see the data presented in Fig. 3 below). This centrality dependence (natural for the low density limit) implies that we still could be far from the hydro regimeff.

Assuming that at SPS the hydro regime is not reached yet, the observed centrality dependence of elliptic flow would indicate that the physics of the system evolution is different in central and peripheral collisions. Elliptic flow peaks at more peripheral collisions because the central collisions exhibit too little flow compared to that expected from the AGS data scaled with multiplicity. A natural explanation for this would be that peripheral collisions are described by hadronic (re)scatterings (the same as at the AGS in both peripheral and central collisions) while in central collisions partonic physics becomes important. One of the possible mechanisms responsible for the change could be a color percolation occurring at high parton densities in the central collisions and discussed in more detail below.

\section{Discussion}

Summarizing, our view of the overall picture is: at AGS energies, the physics of rescattering which defines the system evolution is hadronic in nature, while at SPS it is the same for peripheral collisions, but for central collisions the physics is likely to be partonic. The partonic picture will remain at RHIC energies, with some extension toward more peripheral

it could be that the $p_{t}$ dependence of $v_{2}$ in the hydro model does not agree with experiment.

${ }^{4}$ One can argue that, taking into account systematic uncertainties, the preliminary SPS data for $v_{2} / \varepsilon$ are consistent with being constant as a function of centrality. In this case it would indeed mean that the system has equilibrated and the hydro regime has been reached. The low absolute strength of the elliptic flow in this case would indicate that the equilibration happens at a rather late time when the spatial anisotropy $\varepsilon$ has decreased due to initial "free streaming". We do not exclude this possibility but must wait for the final SPS data and the coming RHIC data to answer the question. 
collisions. At RHIC equilibration becomes more important, but it is not clear if complete thermalization will be reached. At LHC energies the parton densities could become so high that (partonic) rescattering would lead to dynamical equilibration of the (partonic) system (creation of regions of real QGP) and consequently to a hydro-dynamical type of system evolution.

The above picture for collisions of heavy nuclei implies that the shape of the centrality dependence of elliptic flow would change continuously with beam energy. At AGS, the elliptic flow is peaked at an impact parameter value slightly higher than $R_{A}$, just as prescribed by the low density limit. At SPS energies the peak moves toward more peripheral collisions because possibly the physics of relatively central collisions may have changed from hadronic to partonic, which leads to weaker flow than one would expect taking into account the increased multiplicity. If thermalization is not reached at RHIC, the elliptic flow peak could move back toward mid-central collisions because the physics of the peripheral and central collisions will be the same - partonic rescattering, unlike the situation at SPS when peripheral collisions are driven by the hadronic rescatterings resulting in relatively large flow signal. At even higher energies at LHC, the elliptic flow should peak at more peripheral collisions just as predicted by hydrodynamic calculations.

The schematic overall picture based on these observations is presented in Fig. 3, where the ratio of elliptic flow to the initial space elliptic anisotropy is presented as a function of initial particle density.] In this plot we use the experimental charged particle multiplicity, as-

\footnotetext{
${ }^{5}$ At the moment this plot is qualitative as many things shown have large uncertainties. The hydro limits can depend slightly on the initial particle density [1],9] and, more importantly, on the time of thermalization of the system. The values shown are an average of the results of [1,9]. The predictions for the case without QGP are only for the EoS of a massless pion gas. Resonances can soften the EoS and lead to weaker flow. The uncertainty in the experimental points is mainly from the determination of the collision centrality required for calculation of the initial space elliptic
} 
suming that it is proportional to the total particle multiplicity and also to the initial particle multiplicity. For the experimental values we use $d N_{c h} / d y$ at mid-rapidity from [14, 15].

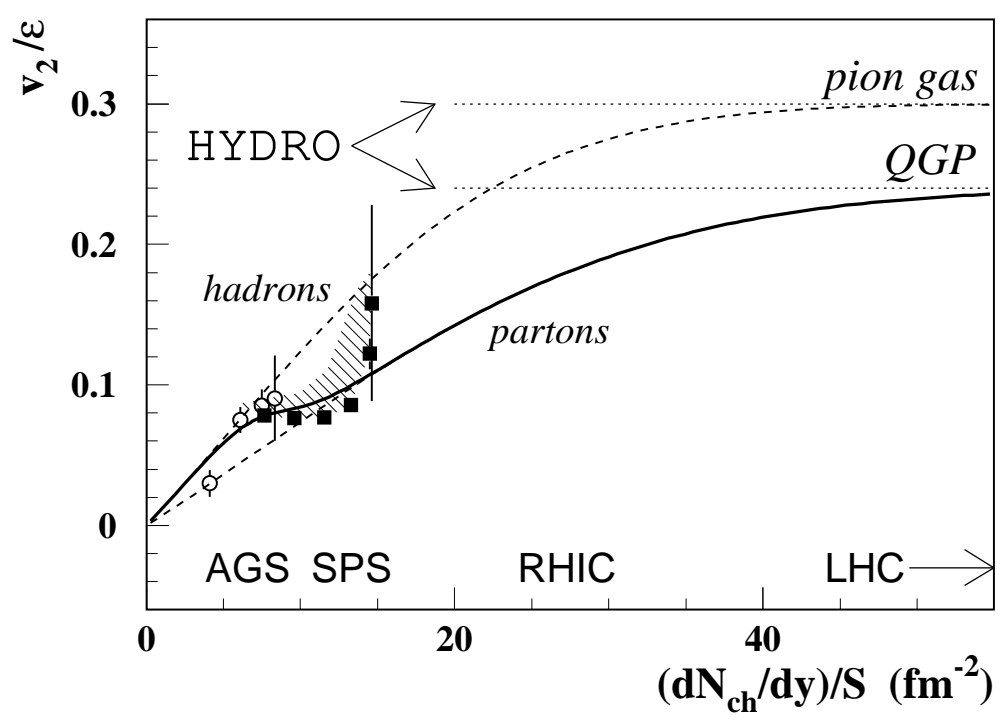

FIG. 3. Elliptic flow divided by the initial space elliptic anisotropy at the AGS (open circles) and the SPS (filled squares). The shaded area shows the uncertainty in the SPS experimental data due to the uncertainty in the centrality determination. See text and footnote for the description of the curves and hydro limits.

In the limit of very low density the objects which rescatter must be hadrons. At some critical density a partial deconfinement happens. Parton density becomes high enough such that the color parton can propagate in the perpendicular plane without hadronization. Each parton is always close enough to other partons which screen its color 0 . Once the motion in

anisotropy and the area of the overlapping region. The data points correspond to the centrality determined from the fraction of the total cross section corresponding to each centrality bin. Higher centralities were estimated from experimental measurement of the number of participants [14]. Finally, the smooth dashed curves are just schematic illustrations for hadronic and partonic scenarios and the solid curve includes a transition between the two.

${ }^{6}$ This picture is very close to the deconfinement (color percolation) model discussed by Satz 16] 
the perpendicular plane becomes easier (there is no need for hadronization), the elliptic flow decreases. Note that the system still can be far from being dynamically thermalized, which would occur only at even higher particle densities. Even more important, such a significant change in the behavior of $v_{2} / \varepsilon$ can only happen if the system is not thermalized. See also the discussion of this question in [6.9] along with the discussion of the possibility of observation of the QGP to hadron gas phase transition.

To prove or disapprove the picture described above one needs more accurate data on the centrality dependence of elliptic flow. We would like to emphasize the importance of flow measurements not only at medium impact parameters but in the full range of centrality including rather central collisions where the anisotropic flow is small. The measurement of elliptic flow and its centrality dependence at RHIC thus becomes very important. Different models predict different rapidity densities for RHIC and LHC. Assuming that they are higher than at SPS by factors of 2 and 8 , respectively, we have indicated the regions expected for $\mathrm{Au}+\mathrm{Au}(\mathrm{Pb}+\mathrm{Pb})$ collisions in Fig. 3. The measurements of elliptic flow in collisions of lighter systems (e.g. $\mathrm{Cu}+\mathrm{Cu}$ ) are also very important since they would cover the region of the SPS $\mathrm{Pb}+\mathrm{Pb}$ data and would be useful in testing the above picture. The new SPS data taken at $40 \mathrm{GeV} \cdot \mathrm{A}$ energy are also of great interest since they would bridge the two other sets of data and may scan the onset of deconfinement from hadronic to partonic physics.

Note that our picture of nuclear collisions and QGP production is different from what is usually discussed, which assumes thermal equilibrium even at rather low beam energies, when QGP is not expected, and then with an increase in collision energy, formation of regions of QGP. We believe that what could happen is that the deconfinement can occur before dynamical thermalization is achieved [17] and that the centrality dependence of elliptic flow would be a good indicator of this.

We are grateful to J.-Y. Ollitrault, U. Heinz, H. Heiselberg, G. Cooper, P. Seyboth, for $J / \Psi$ suppression. 
R. Snellings, H. Sorge, and H.G. Ritter for useful discussions.

This work was supported by the Director, Office of Energy Research, Office of High Energy and Nuclear Physics, Division of Nuclear Physics of the U.S. Department of Energy under Contracts DE-AC03-76SF00098 and DE-FG02-92ER40713.

[1] J.-Y. Ollitrault, Phys. Rev. D 46, 229 (1992).

[2] E877 Collaboration, J. Barrette et al., Phys. Rev. C 55 (1997) 1420.

[3] NA49 Collaboration, H. Appelshäuser et al., Phys. Rev. Lett. 80, 4136 (1998).

[4] S. Voloshin and Y. Zhang, Z. Phys. C 70, 665 (1996); A.M. Poskanzer and S.A. Voloshin, Phys. Rev. C 58, 1671 (1998).

[5] H. Heiselberg and A.-M. Levy, Phys. Rev. C 59, 2716 (1999).

[6] H. Sorge, Phys. Rev. Lett. 82, 2048 (1999).

[7] P. Jacobs and G. Cooper, STAR Note SN402 (1999); http://www.star.bnl.gov/STAR/sno/sno.html

[8] J.-Y. Ollitrault, Nucl. Phys. A638, 195c (1998) ; J.-Y. Ollitrault, talk at QM99, Torino, 1999.

[9] P. Kolb, J. Sollfrank, U. Heinz, Phys. Letters B459, 667 (1999).

[10] H. Sorge, Phys. Rev. C 52, 3291 (1995).

[11] P. Filip, Acta Physica Slovaca, 47, 53 (1997).

[12] W.-C. Chang, PhD thesis, SUNY Stony Brook, 1998; http://www-aix.gsi.de/ misko/e877/theses/theses.htm]

[13] A.M. Poskanzer and S.A. Voloshin for the NA49 Collaboration, talk at QM99, Torino, 1999. The data presented are integrated over rapidity but the values at mid-rapidity are nearly the same. 
[14] G. Cooper for the NA49 Collaboration, talk at QM99, Torino, 1999.

[15] E877 Collaboration, J. Barrette et al., Phys. Rev. C 51 (1995) 3309.

[16] H. Satz, talk at QM99, Torino, 1999; preprint hep-ph/9908339.

[17] R. Stock, Phys. Lett. B456, 277 (1999). 